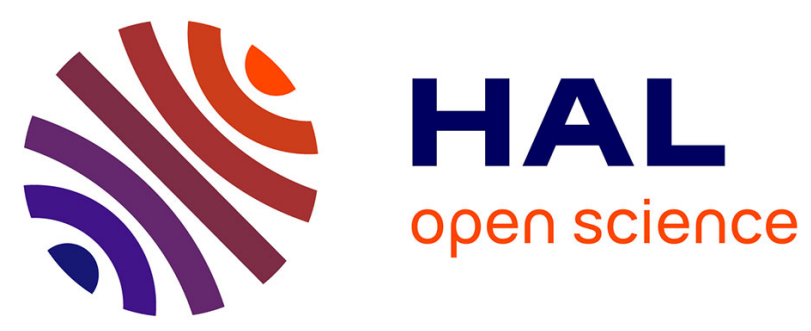

\title{
Vehicles Platooning in Urban Environment: Consensus-based Longitudinal Control with Limited Communications Capabilities
}

Ahmed Khalifa, Olivier Kermorgant, Salvador Dominguez, Philippe Martinet

\section{- To cite this version:}

Ahmed Khalifa, Olivier Kermorgant, Salvador Dominguez, Philippe Martinet. Vehicles Platooning in Urban Environment: Consensus-based Longitudinal Control with Limited Communications Capabilities. International Conference on Control, Automation, Robotics and Vision, Nov 2018, Singapore, Singapore. hal-01866884

\section{HAL Id: hal-01866884 \\ https://hal.science/hal-01866884}

Submitted on 3 Sep 2018

HAL is a multi-disciplinary open access archive for the deposit and dissemination of scientific research documents, whether they are published or not. The documents may come from teaching and research institutions in France or abroad, or from public or private research centers.
L'archive ouverte pluridisciplinaire HAL, est destinée au dépôt et à la diffusion de documents scientifiques de niveau recherche, publiés ou non, émanant des établissements d'enseignement et de recherche français ou étrangers, des laboratoires publics ou privés. 


\title{
Vehicles Platooning in Urban Environment: Consensus-based Longitudinal Control with Limited Communications Capabilities
}

\author{
Ahmed Khalifa, Olivier Kermorgant, Salvador Dominguez, and Philippe Martinet
}

\begin{abstract}
In this research, a general control framework for platooning in urban environment is proposed. A consensusbased control law is described taking into account the nature of traveling in urban environment, that is the human driven leader travels with variable velocity. In addition, the proposed control law does not depend on the predecessor velocity, which in turn allows us to utilize a low cost limited bandwidth communication module by using a sensor-based link for predecessor distance and a communication-based link for leader's information. A constant-spacing policy is used to get a high capacity flow of vehicles. The control system is analyzed and conditions for both internal and string stability are set. The efficiency of the proposed framework and control law is verified via numerical analysis.
\end{abstract}

\section{INTRODUCTION}

Shared transportation systems in urban environments are the current trend to overcome the transportation problems toward an eco-friendly city. One of the recent trends is to use a car-sharing system. However, one of the main problems of the car-sharing system is related to the relocation/redistribution strategies such that the cars are always available and well distributed in all stations. These relocation strategies require more sophisticated techniques to be implemented on cities. An alternative is to guide the cars to move in platoon to relocate them in the available stations.

One of the first and most popular platooning application is developed within the California Partners for Advanced Transit and Highways (PATH) program [1]. Other projects are also proposed in $[2,3]$ that address the problem of platooning in Highways. Unlike the Highways environment, In urban environments each vehicle nevertheless whole the vehicles in the platoon intend to follow a path. Thus, for vehicle control, localization has to be with respect to a path to be followed. So, the path coordinates can be used instead to represent the vehicle model and control law.

The VALET project proposes a novel approach for solving car-sharing vehicles redistribution problem using vehicle platoons guided by professional drivers who comes to pick up and drop off vehicles over the stations.

The authors are with the Laboratoire des Sciences du Numérique de Nantes (LS2N), Ecole Centrale de Nantes, 1 rue de la Noë, 44321 Nantes, France, ahmed.khalifadec-nantes.fr, olivier.kermorganteec-nantes.fr,

salvador.dominguez-qui jadadec-nantes.fr, philippe.martinetdec-nantes.fr.

A. Khalifa is on leave from the Department of Industrial Electronics and Control Engineering, Faculty of Electronic Engineering, Menoufia University, Egypt, ahmed.khalifa@el-eng.menofia.edu.eg.

P. Martinet is with the Inria Sophia Antipolis, 06902 Sophia Antipolis, France, philippe.martineteinria.fr.
In general, the global architecture of platooning has main three layers, including Management, Communication, and Guidance layers. The Guidance layer consists of two sublayers: Intelligent Sensing sub-layer that is a set of sensors and algorithms to determine the vehicle's actual state and Vehicle Control sub-layer that brings the vehicle at the desired states, which consists of two modules, including Longitudinal control and lateral control modules. Each of them has two sub-modules, including Upper-Level Controller (ULC) and Lower-Level Controller (LLC). In this study, we are interested in designing the Longitudinal ULC that provides the desired vehicle linear acceleration that the car has to follow by the Longitudinal LLC to achieve the desired longitudinal states.

The vehicle longitudinal dynamics are inherently nonlinear. For platoon modeling, different models are used in the literature, including linear and nonlinear models. The linear models are frequently used. Several linear models are commonly used. First, single integrator model, which is the simplest one and it is widely different from the real vehicle dynamics [4]. The second model is the double integrator model [5]. The third approach is to use a third order model by adding another state to model approximately the input/output behavior of the power-train which adds complexity to the control design and analysis [6]. To the best of our knowledge, despite huge amount of relevant literature to date, few studies in the literature have handled the problem of platooning in the urban environment/path coordinates, for instance in [7]. In these studies, the authors uses the first order model of the platoon which has some limitations as it is mentioned before. Therefore, in this research, a second order longitudinal dynamic model is used and its corresponding one in the path coordinates is derived such that one can design a controller to achieve control objectives in the path (operational) coordinates.

The simplest platoon controller is proposed in [8] that is called Adaptive Cruise Control (ACC), which controls only the vehicles gas and brake throttle, to maintain a safe distance with the front vehicle. Recently, platoon control is based on a distributed controller known as Cooperative Adaptive Cruise Control (CACC) [9]. In the literature there are four main approaches in designing a platoon controller, including Linear, Optimal, $\mathcal{H}_{\infty}$-based, Sliding Mode, Model predictive, and Consensus-based controller. Different types of Linear Controllers (LC) are widely used in the literature [10]. Some studies propose a sliding mode controller to achieve the string stability of platoon as in [11]. In [12], $\mathcal{H}_{\infty}$ controller is proposed to achieve system robustness. 
The last type is what is called consensus-based controller. Consensus problems of multi-agent systems have attracted an ever-increasing interest in the control community due to their great potential in various applications such as cooperative unmanned air vehicles, automated highway systems scheduling, air traffic control, sensor networks [13]. Recently, CACC control problems using consensus-based algorithms have a tremendous surge of interest among researchers and various algorithms have been developed. Several studies in the literature propose a consensus-based control for platooning in Highways applications as in [14] for example. However, non of theses works consider the control in the path coordinates. In addition, they assume that the leader travel with constant velocity that is not applicable to platooning in urban environment in which, the leader is driven by human, so it may travel with variable velocities. Thus, in this study, the authors propose a controller that can achieve asymptotic stability of the tracking errors and the platoon string stability as well even if the leader travels with variable velocity.

The Information Flow Topology (IFT) represents the Intervehicles communication topology that the vehicles can utilize to acquire the information from its surrounding vehicles. It has a significant impact on the platoon performance. Thanks to the rapid advancement of vehicle-to-vehicle (V2V) communication technology, various IFTs are developed, including Predecessor Following (PF), Predecessor - Leader Following (PLF), Bidirectional (BD), etc. Several approaches have been proposed to improve string stability of a platoon. One of them is to broadcast the leader information to every following vehicle, resulting in the PLF topology [15]. This approach is commonly used in the literature in which the leader communicates with all the vehicles in broadcast, and every other vehicle also considers information from its predecessor to compute the control action. However, for low coast communication solution, due to limited bandwidth capabilities of the on-board communication module two channel communication is not feasible. Thus, in this study, the proposed control law depends on a hybrid PLF topology. That is, the leader broadcasts its information (position, velocity, and acceleration) to all the vehicles via a communicationbased link. For the inter-followers communication, the intervehicle distance is measured by a distance sensor, e.g., laser, i.e., by a sensor-based link. In addition, the proposed control law does not depend on the predecessor velocity.

To sum up, the main contributions of this research are,

- developing a second-order longitudinal platoon control framework in the path coordinates;

- designing a consensus-based controller that is applicable to both motion in urban environment and limited communication capabilities of the vehicles;

- driving conditions for the platoon stability (internal and string).

\section{PROBLEM STATEMENT}

\section{A. Car-like Vehicle Kinematic Model}

Simplifying the car-vehicle system model to a kinematic bicycle model is a common approximation [16]. Consider a car-like vehicle shown in Fig. 1. The kinematic bicycle model combines the left and right wheels into a pair of single wheels at the center of the front and rear axles. The wheels are assumed to have no lateral slip and only the front wheel is steerable.

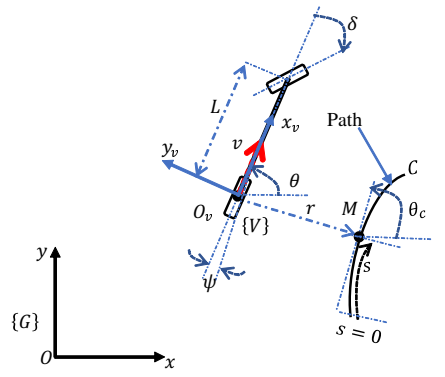

Fig. 1: Kinematic model in Cartesian \& Curvilinear coordinates.

1) Cartesian Coordinates Model: Let $\{V\}, O_{v}-x_{v} y_{v}$, denotes the vehicle body-fixed reference frame with its $x$ axis in the vehicles forward direction and its origin at the center of the rear axle, $O_{v}$, see Fig. 1. The configuration of the vehicle, with respect to the world-fixed inertial reference frame, $\{G\}, O-x y$, is represented by the generalized coordinates $z=\left[\begin{array}{lll}x & y & \theta\end{array}\right]$, where its position is given by $p=\left[\begin{array}{ll}x & y\end{array}\right]^{T} \in \mathbb{C}$ where $\mathbb{C} \subset S E(2)$, while its orientation in the global frame is represented by $\theta . \delta$ is the steering angle in the body frame. The vehicles velocity is by definition $v$ in the vehicles $\mathrm{x}$-direction, $x_{v}$, and zero in the y-direction, $y_{v}$, since the wheels cannot slip sideways. If the front wheel is located at distance $L$ from the rear wheel along the orientation of the vehicle, then the kinematic model is given by

$$
\begin{aligned}
\dot{x} & =v \cos (\theta), \\
\dot{y} & =v \sin (\theta), \\
\dot{\theta} & =\frac{v}{L} \tan (\delta) .
\end{aligned}
$$

2) Path Coordinates Model: Let us define the curvilinear coordinates $(s r \psi)^{T}$, as shown in Fig. 1. The tracking path, $C$, defined in the Global Frame, can be represented as a function of its length $s$ (Curvilinear abscissa) at the closest point $M$ to $O_{v}$, the relative angle (angular deviation), $\psi=$ $\theta-\theta_{c}$, of the vehicle with respect to the path, where $\theta_{c}$ is the angle between the path tangent at $M$ and the $x$-axis, and finally, the lateral distance (lateral deviation), $r$, which is the signed orthogonal distance from the center of the rear axle, $O_{v}$, to the closest point on the path, $M$. From the geometry in Fig. 1, the kinematic model in the path coordinates is given by

$$
\begin{aligned}
\dot{s} & =v \frac{\cos (\psi)}{1-r \kappa(s)}, \\
\dot{r} & =v \sin (\psi), \\
\dot{\psi} & =v\left(\frac{\tan (\delta)}{L}-\frac{\kappa(s) \cos (\psi)}{1-r \kappa(s)}\right),
\end{aligned}
$$

where $\kappa(s)$ is the curvature of path at point $M$. 
3) Lateral Controller: Motion control of autonomous vehicles moving either alone or in platoon requires design of lateral and longitudinal control to achieve performance objectives in the lateral and longitudinal directions. The lateral controller will provide the desired steering angle as the control signal. The vehicle lateral control will be designed to achieve the lateral control objectives which are: independently from the longitudinal states/controller, $r \rightarrow 0$ and $\psi \rightarrow 0$ as $t \rightarrow \infty$. An interesting and useful method for controlling kinematic models of car-like vehicle systems given by (2) can be found in [17] which is based on the chained forms. In this work, the lateral control law is given by

$$
\begin{array}{r}
\delta=\operatorname{atan}\left(L \left(\frac { \operatorname { c o s } ^ { 3 } ( \psi ) } { ( 1 - r \kappa ) ^ { 2 } } \left(\frac{d \kappa}{d s} r \tan (\psi)-k_{d}(1-r \kappa) \tan (\psi)\right.\right.\right. \\
\left.\left.\left.-k_{p} r+\kappa(1-r \kappa) \tan ^{2}(\psi)\right)+\frac{\kappa \cos (\psi)}{1-r \kappa}\right)\right),
\end{array}
$$

where $k_{p}$ and $k_{d}$ are the controller parameters to be tuned for a desired system response.

\section{B. Car-like Vehicle Longitudinal Dynamic Model}

For each vehicle, several factors determine its longitudinal dynamic behavior, which is inherently nonlinear, including the engine, drive line, brake system, aerodynamics drag, tire friction, rolling distance, and gravitational force, ..., etc [18]. To make a balance between accuracy and conciseness, it is assumed that:

Assumption 1. The vehicle body is considered to be rigid and symmetric.

Assumption 2. The driving and braking inputs are integrated into one control input.

Assumption 3. The influence of pitch and yaw motions is considered to be neglected.

Therefore, the longitudinal dynamic equation can be represented by

$$
M_{v} a+C_{v} v+G_{v}=F_{v}
$$

where $a=\dot{v}$ denotes the vehicle's acceleration in the vehicle frame, and $M_{v}, C_{v}, G_{v}$, and $F_{v}$ are the vehicle's Inertia effect, Coriolis effect, Gravity effect, and input force, respectively.

The inverse model compensation technique is frequently used to eliminate the non-linearities in longitudinal dynamics for the purpose of controller design [19].

The control law of the inverse dynamics technique is given by

$$
F_{v}=M_{v} \mu+C_{v} v+G_{v}
$$

where $\mu$ is the new input signal after system linearization which has to be designed such that the control objectives can be achieved. By applying this lower-level controller, one can obtain a linear model for vehicle longitudinal dynamics as

$$
a=\mu,
$$

Most of the research consider the Cartesian coordinates for control design. However, as we intended to platoon in the urban environment, then the relation between the dynamics in the path and Cartesian coordinates will be derived such that the controller can be designed in the path coordinates.

Let us recast $(2 a)$ as

$$
\dot{s}=T v
$$

where $T$ is given by

$$
T=\frac{\cos (\psi)}{(1-r \kappa)} .
$$

Vehicle's acceleration in the path coordinates can be found from the differentiation of (7) with respect to (w.r.t) time as follows

$$
\eta=T a+\dot{T} v
$$

where $\eta=\dot{q}=\ddot{s}$ and $q=\dot{s}$ are the vehicle's acceleration and velocity in the path coordinates respectively.

Consequently, if the mapping from the control signal (i.e., desired acceleration) in the path coordinates, $u$, to that in the Cartesian coordinates, $\mu$, is given by

$$
\mu=\frac{1}{T}(u-\dot{T} v),
$$

then, the vehicle longitudinal dynamic model in the path coordinates can be represented by

$$
\begin{aligned}
& \dot{s}=q, \\
& \dot{q}=\eta=u .
\end{aligned}
$$

\section{Platoon Longitudinal Model}

From a control viewpoint, the main goal is to create the platoon and then maintain a spacing policy in the presence of perturbations. Consider $N+1$ vehicles are required to move in a platoon, as illustrated in Fig. 2, including a leading vehicle (noted as the leader with index 0 ) and $N$ following vehicles (noted as followers with index $i ; i=1,2, \ldots, N$ ). The platoon runs on urban and flat road, and the vehicles can share information either sensor-based or communicationbased way. All the vehicles are assumed to have equal

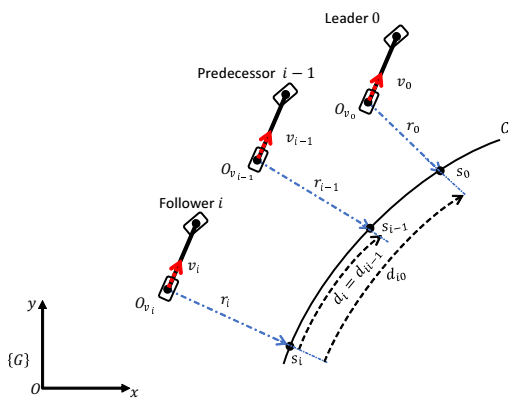

Fig. 2: Platoon representation in the path coordinates.

dynamics. The position, velocity, acceleration, and control 
input (i.e., desired acceleration.) of vehicle $i$ are denoted with $s_{i}, q_{i}, \eta_{i}$, and $u_{i}$ in the path coordinates. Consequently, the dynamics can be written in the path coordinates as:

$$
\begin{aligned}
& \dot{s}_{i}=q_{i}, \\
& \dot{q}_{i}=u_{i},
\end{aligned}
$$

where the transformation between the two coordinates can be implemented via (2a) and (10).

For the string of vehicles, the inter-vehicle distance, $d_{i}=$ $s_{j}-s_{i}$, is the actual curvilinear distance between vehicle $i$ and its predecessor $j=i-1$, and $d_{r, i}$ is the desired inter-vehicle distance between vehicles $i$ and $j$. Several spacing policies are proposed in the literature [20, 21]. For high capacity, small vehicle to vehicle spacing, the constant spacing policy method is utilized.

\section{Control Objectives}

The platoon has to travel in urban environment (curvilinear path) and track a leader that is either autonomous or manually driven, and each follower vehicle, $i$, has the following information:

- position, velocity, and acceleration of the leader, $s_{0}, q_{0}$, $\eta_{0}$ via communication-based link;

- position of the front vehicle, i.e., its predecessor $s_{i-1}$ via sensor-based link due to the limited communication capability of the on-board communication module.

Under these conditions, $\forall i=1, \ldots, N$, the following goals have to be achieved:

- Asymptotic stability of the tracking errors.

- String stability.

\section{CONTROL DESIGN}

The information flow structure among platoon can be modeled by a graph in which each vehicle is a node whose dynamics are described by (12). The information flow among followers can be modeled by a directed graph (digraph) $\mathcal{G}=(\mathcal{V}, \mathcal{E}, \mathcal{M})$ characterized by the set of nodes $\mathcal{V}=$ $1, \ldots, N$ and set of edge $\mathcal{E} \subseteq \mathcal{V} \times \mathcal{V}$. Each edge represents a directional information exchange between two vehicles. The topology of the graph is described by an adjacency matrix $\mathcal{M}=\left[m_{i j}\right]_{N \times N}$, in which the element $m_{i j}=1$ if vehicle $i$ can obtain information from vehicle $j$; otherwise, $m_{i j}=0$. Also, the self-edges are not allowed, i.e., $m_{i i}=0$. To include the leader (with index 0) in the network, an augmented digraph $\overline{\mathcal{G}}$ is used.

Authors propose the following control law

$$
u_{i}=\eta_{0}+b\left(q_{0}-q_{i}\right)+\sum_{j=0}^{N} k_{i j} m_{i j}\left(s_{j}-s_{i}-d_{r, i j}\right) \text {, }
$$

where, $b$ and $k_{i j}$ are tuning parameters, $d_{r, i i-1}$ is given by

$$
d_{r, i i-1}=l_{f, i}+d_{b b, i i-1}+l_{r, i-1},
$$

where $l_{f, i}$ and $l_{r, i}$ is the distance from the front and rear bumpers to the rear axle center, $O_{v_{i}}$, of vehicle $i$, respectively, and $d_{b b, i i-1}$ is the desired constant space between vehicle $i$ and its predecessor (bumper-to-bumper distance).
For the Predecessor - Leader Following (PLF) information flow topology, where the leader communicates with all the vehicles in broadcast, and every other vehicle also considers information from its predecessor, (13) can be recast as

$$
u_{i}=\eta_{0}+b e_{q, i 0}+k_{0} e_{s, i 0}, \quad \forall i=1,
$$

and

$$
u_{i}=\eta_{0}+b e_{q, i 0}+k_{0} e_{s, i 0}+k_{1} e_{s, i}, \quad \forall i>1,
$$

where $e_{s, i 0}=s_{0}-s_{i}-d_{r, i 0}, e_{q, i 0}=q_{0}-q_{i}, e_{s, i}=s_{j}-$ $s_{i}-d_{r, i}$, for $k_{i 0}$; assume $k_{10}=k_{20}=\cdots=k_{N 0}=k_{0}$, and for $k_{i j}$; assume $k_{21}=k_{32}=\cdots=k_{N N-1}=k_{1}$.

Theorem 1. Consider a $N$ car-like vehicle platoon following a leader, the longitudinal model expressed as (1), (2), (4 - 10), and (12), with assumptions 1 - 3, and the control algorithm described in (15) and (16) is applied to it. Then, the system is asymptotically stable and the platoon is strong string stable as long as the following conditions are satisfied: $\forall \gamma<1, b>0, c=0.25 b^{2}, k_{1}=\gamma c$, and $k_{0}=(1-\gamma) c$.

Proof. Firstly, the internal stability analysis is done as follows.

Applying the control law (16) to the system (12), and if one define the inter-vehicle distance error, $e_{s, i}$, in terms of the errors with leader, $e_{s, i 0}$, as $e_{s, i}=s_{j}-s_{i}-d_{r, i}=e_{s, i 0}-$ $e_{s, j 0}, d_{r, i}=d_{r, i 0}-d_{r, j 0}, j=i-1$, then the error dynamics in terms of the error with leader is given by

$$
\begin{aligned}
& \dot{e}_{s, i 0}=e_{q, i 0}, \\
& \dot{e}_{q, 10}=-b e_{q, 10}-k_{0} e_{s, 10}, \\
& \dot{e}_{q, i 0}=-b e_{q, i 0}-\left(k_{0}+k_{1}\right) e_{s, i 0}+k_{1} e_{s, j 0}, \forall i>1 .
\end{aligned}
$$

Let us define $e_{s}=\left[e_{s, 10}, \ldots, e_{s, i 0}, \ldots, e_{s, N 0}\right]^{T}$ and $e_{q}=$ $\left[e_{q, 10}, \ldots, e_{q, i 0}, \ldots, e_{q, N 0}\right]^{T}$ as the position and speed error vectors, respectively, then the error state vector, $\mathcal{X}(t)=$ $\left[\begin{array}{ll}e_{s} & e_{q}\end{array}\right]^{T}$, is given by

$$
\dot{\mathcal{X}}(t)=A \mathcal{X}(t)
$$

where $A$ is described as follows

$$
A=\left[\begin{array}{cc}
O_{N} & I_{N} \\
-P & -D
\end{array}\right]
$$

where $I_{n}$ and $O_{n}$ denote $(n \times n)$ identity and $(n \times n)$ null matrices, respectively, $D=\operatorname{diag}\{b, \ldots, b\} \in \mathbb{R}^{N \times N}$, and $P=\left[p_{i i}\right] \in \mathbb{R}^{N \times N}$ is represented by

$$
p_{i i}= \begin{cases}k_{0}, & j=i, i=1, \\ k_{0}+k_{1}, & j=i, i>1, \\ -k_{1}, & j=i-1, i>1, \\ 0, & \text { otherwise. }\end{cases}
$$

The eigenvalues of $P, \lambda_{p, i}$, can be calculated as

$$
\lambda_{p, i}= \begin{cases}k_{0}, & i=1, \\ k_{0}+k_{1}, & i>1 .\end{cases}
$$

Thus, $\forall k_{0}>0$ and $k_{1}>0$, the matrix $P$ is positive stable. In addition, $\forall b>0$, the matrix $D$ is positive stable. 
Lemma 1. Schur's formula [22]. Let matrices $\mathcal{F}_{11}, \mathcal{F}_{12}, \mathcal{F}_{21}, \mathcal{F}_{22} \in \mathbb{R}^{N \times N}$ and $\mathcal{M}=\left[\begin{array}{ll}\mathcal{F}_{11} & \mathcal{F}_{12} \\ \mathcal{F}_{21} & \mathcal{F}_{22}\end{array}\right]$. If $\mathcal{F}_{11}, \mathcal{F}_{12}, \mathcal{F}_{21}, \mathcal{F}_{22}$ commute pairwise, i.e., $\mathcal{F}_{r s} \mathcal{F}_{n m}=$ $\mathcal{F}_{n m} \mathcal{F}_{r s}$ for all possible pairs of indices $r, s$ and $n, m$, then the determinate of $\mathcal{M}$, $\operatorname{det}(M)=\operatorname{det}\left(\mathcal{F}_{11} \mathcal{F}_{22}-\mathcal{F}_{12} \mathcal{F}_{21}\right)$

Lemma 2. [23]. Let a complex coefficient polynomial, $f(z)=z^{2}+(a+i b) z+(c+i d)$, where $a, b, c$, and $d \in \mathbb{R} . f(z)$ is Hurwtiz stable if and only if $a>0$ and $a b d+a^{2} c-d^{2}>0$.

According to Lemma 1, the characteristics polynomial of $A$ can be calculated as follows:

$$
\begin{aligned}
\operatorname{det}\left(S I_{2 N}-A\right) & =\operatorname{det}\left(\left[\begin{array}{cc}
S I_{N} & -I_{N} \\
P & S I_{N}+D
\end{array}\right]\right) \\
& =\operatorname{det}\left(S^{2} I_{N}+b I_{N} S+P\right) \\
& =\prod_{i=1}^{N}\left(S^{2}+b S+\lambda_{p, i}\right) .
\end{aligned}
$$

The polynomial $S^{2}+b S+\lambda_{p, i}$ is Hurwitz stable, according to Lemma 2 and the conditions $b>0, k_{0}>0, k_{1}>0$. This implies that all eigenvalues of $A$ have negative real parts. Consequently, the error dynamics is asymptotically stable i.e., $\lim _{t \rightarrow \infty} \mathcal{X}(t)=0$.

Secondly, the string stability analysis is done as follows. For simplicity, let us define the following errors $e_{i}=s_{j}-$ $s_{i}-d_{r, i}, \dot{e}_{i}=q_{j}-q_{i}, \ddot{e}_{i}=u_{j}-u_{i}, e_{i 0}=s_{0}-s_{i}-d_{r, i 0}$, and $\dot{e}_{i 0}=q_{0}-q_{i}$.

Form (16) one can write $\ddot{e}_{i}$ as

$$
\begin{aligned}
\ddot{e}_{i} & =u_{j}-u_{i} \\
& =b \dot{e}_{j 0}+k_{0} e_{j 0}+k_{1} e_{j}-b \dot{e}_{i 0}-k_{0} e_{i 0}-k_{1} e_{i},
\end{aligned}
$$

Writing (23) in the S-domain results in

$$
\begin{aligned}
S^{2} E_{i}(S) & =b S E_{j 0}(S)+k_{0} E_{j 0}(S)+k_{1} E_{j}(S) \\
& -b S E_{i 0}(S)-k_{0} E_{i 0}(S)-k_{1} E_{i}(S) .
\end{aligned}
$$

Substituting with $e_{i 0}=e_{i}+e_{j 0}$ or $E_{i 0}(S)=E_{i}(S)+E_{j 0}(S)$ into (24), then the transfer function $H(S)=\frac{E_{i}(S)}{E_{j}(S)}$ can be defined as

$$
H(S)=\frac{k_{1}}{S^{2}+b S+c},
$$

where $c=k_{0}+k_{1}$.

Conditions for string stability:

- In time domain, for strong string stability: $\|h(t)\|_{1}<1$

- In frequency domain

- for strong string stability: $\|H(S)\|_{\infty}<1$ and $h(t)>0$

The poles of $H(S)$, second order system, are $p_{1,2}=$ $-0.5 b \pm \sqrt{(0.5 b)^{2}-c}$

To achieve $h(t)>0$, the response of $H(S)$ has to be overdamped, i.e., $(0.5 b)^{2}>c$, or critical-damped, i.e., $(0.5 b)^{2}=$ $c$. However, for faster response the parameters of $H(S)$ are chosen for a critical-damped response, i.e., $(0.5 b)^{2}=c$. In this case, the impulse response is represented by

$$
h(t)=k_{1} t e^{-0.5 b t} .
$$

Thus, $\forall k_{1}>0$, one can obtain $h(t)>0$.

Lemma 3. [24]. If $F(s)$ is a stable, proper transfer function, with $|F(0)|=\beta$, and if $f(t)=\mathcal{L}^{-1}\{F(s)\}$ is its impulse response, then $\|h(t)\|_{1}=\beta$ if and only if $h(t)$ does not change sign.

According to Lemma 3,

$$
\begin{aligned}
\|h(t)\|_{1} & =|H(0)| \\
& =\frac{k_{1}}{c}=\gamma,
\end{aligned}
$$

Thus, by choosing $k_{1}=\gamma c$ with $\gamma<1$, then $\|h(t)\|_{1}<1$ and as a result the platoon is strong string stable.

\section{RESULTS}

\section{A. Environment}

The previously proposed control strategy is simulated in MATLAB/SIMULINK program for the considered Urban cars platooning. We consider a platoon composed of a homogeneous 4 vehicles plus a manually driven leader. For emulation of motion in urban environment, the trajectory shown in Fig. 3, is used as a reference trajectory to be followed by all the cars. The vehicles are initially parked near the reference path with different initial poses. The lateral controller (3) is in charge of producing steering angle of the vehicle. Gains of the lateral control law are tuned in order to get a satisfactory response of the lateral and angular deviations, see Fig. 3 for the results. The path tracking capability of the vehicle is enlightened in Fig. 3. The selected lateral control parameters are $k_{p}=200$ and $k_{d}=95$.

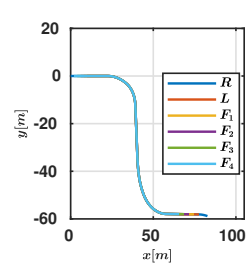

(a) Trajectory Tracking

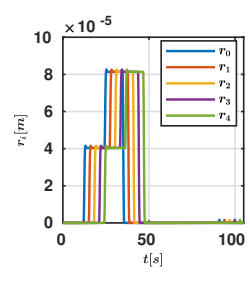

(b) $r_{i}$

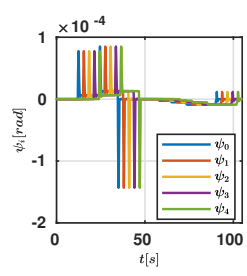

(c) $\psi_{i}$
Fig. 3: Lateral Control Results: $R$ : Reference Trajectory, $L$ : Leader Actual Trajectory, and $F_{i}$ : Follower $i$ Actual Trajectory.

The longitudinal controller is implemented at rate of $100 \mathrm{~Hz}$ assuming that the vehicle states are available at this rate. Control parameters are selected to guarantee the internal stability and string stability. The selected control parameters are reported in Table I.

\section{B. Normal Platooning}

Fig. 4 shows the results for the normal consensus scenario. The leader travels with constant velocity. The results confirm the capability of the proposed approach of creating and maintaining the platoon in case of leader travel with constant velocity. The vehicles are starting from distances different 
TABLE I: Simulation Parameters

\begin{tabular}{|c|c|c|c|}
\hline Parameter & Value & Parameter & Value \\
\hline$L$ & $1 \mathrm{~m}$ & $a_{\max }$ & $1 \mathrm{~m} / \mathrm{s}^{2}$ \\
\hline$a_{\min }$ & $-3 \mathrm{~m} / \mathrm{s}^{2}$ & $v_{\max }$ & $8 \mathrm{~m} / \mathrm{s}$ \\
\hline$v_{\min }$ & $0 \mathrm{~m} / \mathrm{s}$ & $b$ & 1.6 \\
\hline$d_{r, i}$ & $3 \mathrm{~m}$ & $\gamma$ & 0.1 \\
\hline
\end{tabular}

from the one required by the spacing policy and reach the consensus and the curvilinear position and speed errors converge asymptotically. Moreover, the followers track the leader zero acceleration (i.e., the control effort reaches zero after consensus).

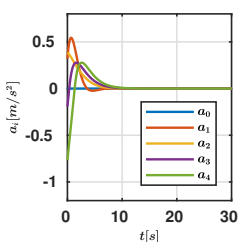

(a) $a_{i}$

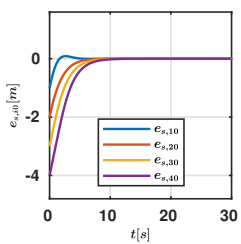

(d) $e_{s, i 0}$

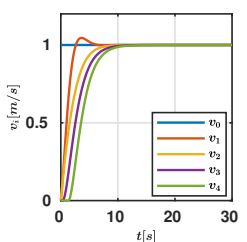

(b) $v_{i}$

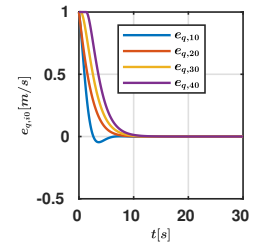

(e) $e_{q, i 0}$

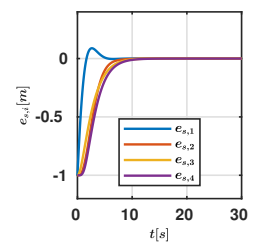

(c) $e_{s, i}$

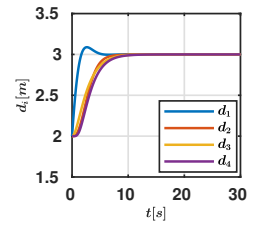

(f) $d_{i}$
Fig. 4: Normal Platooning Longitudinal Control Results

\section{Variable Speed Scenario}

Fig. 5 shows the results for the leader tracking consensus scenario. The results confirm the capability of the proposed approach of creating and maintaining the platoon in case of a manually driven leader (i.e., the velocity is not constant). The vehicles are starting from distances different from the one required by the spacing policy and reach the consensus and the curvilinear position and speed errors converge asymptotically. Furthermore, the followers track the leader acceleration that has a trapezoidal shape.

\section{Braking Scenario}

To investigate the controller robustness against different driving scenarios, we test the ability of the proposed strategy in case of the leader sudden braking. Results in Fig. 6 show how the platoon reacts in the case of a braking maneuver performed by the leader from $1.5 \mathrm{~m} / \mathrm{s}$ to a full stop. The results show that the inter-vehicle distance between vehicle 0 and vehicle $1, d_{1}$, suffers an approximately $0.6 \mathrm{~m}$ decrease, while $d_{2}$ suffers an approximately $0.09 \mathrm{~m}$ decrease, and $d_{3}$ and $d_{4}$ is further smaller. These results illustrate that the sudden disturbance on the platoon is attenuated along the rest of the platoon (i.e., string stable platoon).

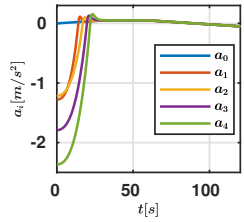

(a) $a_{i}$

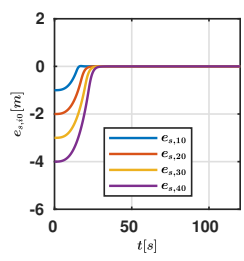

(d) $e_{s, i 0}$

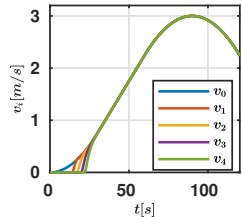

(b) $v_{i}$

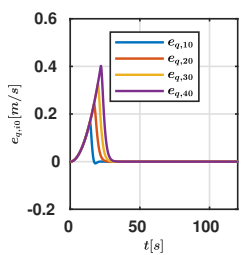

(e) $e_{q, i 0}$

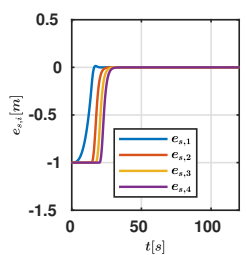

(c) $e_{s, i}$

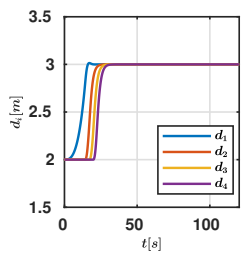

(f) $d_{i}$
Fig. 5: Variable Speed Platooning Longitudinal Control Results

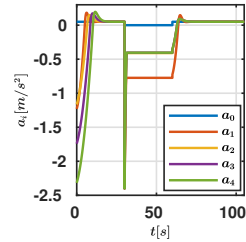

(a) $a_{i}$

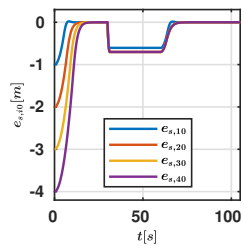

(d) $e_{s, i 0}$

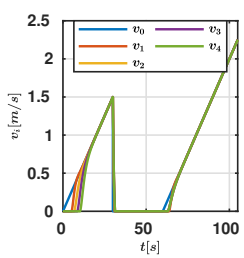

(b) $v_{i}$

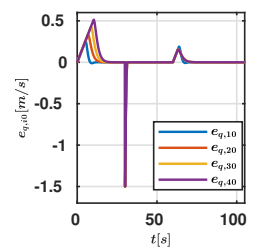

(e) $e_{q, i 0}$

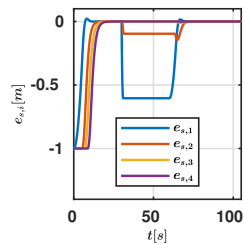

(c) $e_{s, i}$

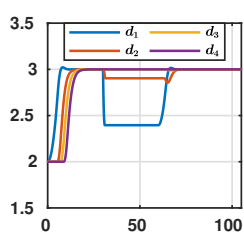

(f) $d_{i}$
Fig. 6: Platooning Longitudinal Control Results: Leader Sudden Brake

\section{CONCLUSIONS}

A consensus-based control technique for platooning in urban environment is proposed. A second-order longitudinal model of the platoon in the path coordinates is driven. General architecture is proposed to design the controller in the path coordinates in order to achieve some objectives defined the path coordinates with a relation that maps the control signals in the Cartesian and path coordinates. The proposed algorithm does't need for the predecessor's velocity, so one can depend only on the distance sensor without the need to communicate with the predecessor's such that lower bandwidth communication module can be utilized. A constant-spacing policy is used to get a high capacity flow of vehicles. The controller is design to achieve zero tracking errors in case the leader is manually driven (i.e., leader travels with variable velocity) to be suited to work in VALET project. Internal stability is analyzed and the asymptomatic 
stability of the tracking errors is proven. Conditions for platoon string stability are driven. Efficiency of the proposed control framework and control algorithm are enlightened via numerical analysis.

\section{ACKNOWLEDGMENTS}

This paper describes a work carried out in the framework of the Valet project, reference ANR-15-CE22-0013-02.

\section{REFERENCES}

[1] Steven E Shladover. "PATH at 20History and major milestones". In: IEEE Transactions on intelligent transportation systems 8.4 (2007), pp. 584-592.

[2] Sadayuki Tsugawa, Shin Kato, and Keiji Aoki. "An automated truck platoon for energy saving". In: IEEE/RSJ International Conference on Intelligent Robots and Systems (IROS). IEEE. 2011, pp. 41094114.

[3] Erik Coelingh and Stefan Solyom. "All aboard the robotic road train". In: Ieee Spectrum 49.11 (2012).

[4] Fu Lin, Makan Fardad, and Mihailo R Jovanovic. "Optimal control of vehicular formations with nearest neighbor interactions". In: IEEE Transactions on Automatic Control 57.9 (2012), pp. 2203-2218.

[5] Maode Yan et al. "Consensus Based Platoon Algorithm for Velocity-Measurement-Absent Vehicles with Actuator Saturation". In: Journal of Advanced Transportation 2017 (2017).

[6] Jeroen C Zegers et al. "Consensus control for vehicular platooning with velocity constraints". In: IEEE Transactions on Control Systems Technology (2017).

[7] Shengbo Eben Li et al. "Minimum fuel control strategy in automated car-following scenarios". In: IEEE Transactions on Vehicular Technology 61.3 (2012), pp. 998-1007.

[8] Hermann Winner et al. Adaptive cruise control system aspects and development trends. Tech. rep. SAE Technical Paper, 1996.

[9] Joel VanderWerf et al. "Modeling effects of driver control assistance systems on traffic". In: Transportation Research Record: Journal of the Transportation Research Board 1748 (2001), pp. 167-174.

[10] Hossein Chehardoli and MR Homaeinezhad. "Thirdorder leader-following consensus protocol of traffic flow formed by cooperative vehicular platoons by considering time delay: constant spacing strategy". In: Proceedings of the Institution of Mechanical Engineers, Part I: Journal of Systems and Control Engineering (2017), p. 0959651817750521.

[11] Yujia Wu et al. "Distributed sliding mode control for multi-vehicle systems with positive definite topologies". In: IEEE $55^{\text {th }}$ Conference on Decision and Control (CDC). IEEE. 2016, pp. 5213-5219.
[12] Yang Zheng et al. "Platooning of connected vehicles with undirected topologies: Robustness analysis and distributed h-infinity controller synthesis". In: IEEE Transactions on Intelligent Transportation Systems 19.5 (2018), pp. 1353-1364.

[13] Toru Namerikawa, Yasuhiro Kuriki, and Ahmed Khalifa. "Consensus-based cooperative formation control for multiquadcopter system with unidirectional network connections". In: Journal of Dynamic Systems, Measurement, and Control 140.4 (2018), p. 044502.

[14] Stefania Santini et al. "A consensus-based approach for platooning with intervehicular communications and its validation in realistic scenarios". In: IEEE Transactions on Vehicular Technology 66.3 (2017), pp. 1985-1999.

[15] DVAHG Swaroop and JK Hedrick. "Constant spacing strategies for platooning in automated highway systems". In: Journal of dynamic systems, measurement, and control 121.3 (1999), pp. 462-470.

[16] Stefan Forrest Campbell. "Steering control of an autonomous ground vehicle with application to the DARPA urban challenge". PhD thesis. Massachusetts Institute of Technology, 2007.

[17] Benoit Thuilot et al. "Accurate automatic guidance of an urban electric vehicle relying on a kinematic GPS sensor". In: IFAC Proceedings Volumes 37.8 (2004), pp. $155-160$.

[18] RM DeSantis. "Path-tracking for car-like robots with single and double steering". In: IEEE Transactions on vehicular technology 44.2 (1995), pp. 366-377.

[19] Yang Zheng et al. "Stability and scalability of homogeneous vehicular platoon: Study on the influence of information flow topologies". In: IEEE Transactions on Intelligent Transportation Systems 17.1 (2016), pp. 14-26.

[20] DVAHG Swaroop et al. "A comparision of spacing and headway control laws for automatically controlled vehicles1". In: Vehicle System Dynamics 23.1 (1994), pp. 597-625.

[21] Kumaragovindhan Santhanakrishnan and Rajesh Rajamani. "On spacing policies for highway vehicle automation". In: IEEE Transactions on Intelligent Transportation Systems 4.4 (2003), pp. 198-204.

[22] Yongcan Cao, Wei Ren, and Yan Li. "Distributed discrete-time coordinated tracking with a time-varying reference state and limited communication”. In: Automatica 45.5 (2009), pp. 1299-1305.

[23] P. C. Parks and V. Hahn. Stability theory. Prentice Hall New York, 1993.

[24] S Darbha. "String stability of interconnected systems: An application to platooning in automated highway systems". PhD thesis. University of California, 1994. 